\title{
Troponin I Type 3 Measurement
}

National Cancer Institute

\section{Source}

National Cancer Institute. Troponin I Type 3 Measurement. NCI Thesaurus. Code

C135448.

The determination of the amount of troponin I type 3 (cardiac muscle) in a biological sample. 